\title{
Paternidad adolescente: significados y prácticas desde una perspectiva socioconstruccionista
}

\author{
María del Pilar Gómez-González, Ph. D. ${ }^{a}$ \\ Fundación Universitaria del Área Andina, Colombia \\ Juan Carlos Ramírez-Rodríguez, Ph. D. ${ }^{\mathrm{b}}$ \\ Centro Universitario de Ciencias Económico-Administrativas, Universidad de Guadalajara, México
}

mariadelpilardrsp@gmail.com

\section{Resumen (analítico)}

La paternidad en la adolescencia tradicionalmente ha sido estigmatizada, llevando a la generación de barreras y desconocimiento de vivencias, motivaciones y significados. Por ello se planteó como objetivo comprender la construcción y significados de la paternidad presente y activa de hombres adolescentes que residen en Guadalajara, Jalisco, México. Se realizó un estudio cualitativo socioconstruccionista en el que participaron hombres de 16 a 19 años que eran padres o que su pareja estaba en embarazo. Se realizaron entrevistas semiestructuradas y observación de redes sociales; se realizó un análisis de contenido. Los resultados dan cuenta de la intersección entre masculinidades y paternidades, demostrando que en las motivaciones para su paternidad se destacan la búsqueda de reconocimiento social, la conformación de una familia y la búsqueda de suplir necesidades emocionales a través de la pareja y del hijo/hija.

\section{Palabras clave}

Paternidad, hombres adolescentes, masculinidades, género, socioconstruccionismo.

\section{Thesauro}

Tesauro de Ciencias Sociales de la Unesco; Tesauro Bireme.

\section{Para citar este artículo}

Gómez-González, M. del P., \& Ramírez-Rodríguez, J. C. (2022). Paternidad adolescente: significados y prácticas desde una perspectiva socioconstruccionista. Revista Latinoamericana de Ciencias Sociales, Niñez y Juventud, 20(1), 1-19. https://dx.doi.org/10.11600/rlcsnj.20.1.4447

\section{Historial}

Recibido: 16.12 .2020

Aceptado: 08.07.2021

Publicado: 01.12.2021

\section{Información artículo}

Este artículo se deriva del proyecto denominado Construcción social de las emociones sobre la paternidad en hombres adolescentes, financiado por la Fundación Universitaria del Área Andina con código CV2017-P37, la Universidad de Guadalajara y por el Consejo Nacional de Ciencia y Tecnología. Realizado entre marzo de 2015 y diciembre de 2018. Área: sociología. Subárea: temas especiales (estudios de género). 


\section{Adolescent fatherhood: meanings and practices from a socio-constructionist perspective}

\section{Abstract (analytical)}

Adolescent paternity has traditionally been stigmatized, which has generated barriers and ignorance of the experiences, motivations and meanings of young fathers. This is why this study has the objective of understanding the construction and meanings of the present and active paternity of adolescent men living in Guadalajara, Mexico. A qualitative socio-constructionist study was carried out with young men aged 16 to 19 who were parents or their partner was pregnant. Semi-structured interviews and observation of their social networks were carried out followed by content analysis. The results of the study highlight the intersections between masculinities and paternities. Notable motivations for their paternity include their desire for social recognition, their interest in forming a family and meeting their emotional needs through their partner and son/daughter.

Keywords

Fatherhood, adolescent men, masculinities, gender, socioconstructionism.

\section{Paternidade na adolescência: significados e práticas na perspectiva socioconstrucionista}

\section{Resumo (analítico)}

A paternidade na adolescência tem sido tradicionalmente estigmatizada, levando à geração de barreiras e ignorância de experiências, motivações e significados, razão pela qual o objetivo era compreender a construção e os significados da paternidade atual e ativa dos adolescentes homens residentes em Guadalajara, Jalisco, México. Foi realizado um estudo sócio-construcionista qualitativo, envolvendo homens de 16 a 19 anos que eram pais ou que o casal estava grávido. Foram realizadas entrevistas semiestruturadas e observação das redes sociais, análise de conteúdo. Os resultados mostram a interseção entre masculinidades e paternidades, demonstrando nas motivações de sua paternidade a busca pelo reconhecimento social, a formação de uma família e a busca de suprir necessidades emocionais por meio do casal e do filho / filha.

\section{Palavras-chave}

Paternidade, homens adolescentes, masculinidades, gênero, sócio-construcionismo.

\section{Información autores}

[a] Optómetra. Magíster en Epidemiología, Universidad del Valle, Colombia. Doctora en Ciencias de la Salud Pública, Universidad de Guadalajara, México. Investigadora-docente, directora posgrados en salud, Fundación Universitaria del Área Andina. (DD) 0000-0002-4048-0008. H5: 4. Correo electrónico: mariadelpilardrsp@gmail.com

[b] Médico, cirujano y partero. Doctor en Ciencias Sociales con especialidad en Antropología Social, Centro de Investigaciones y Estudios Superiores en Antropología Social, Occidente. Postdoctor en Políticas en Salud Reproductiva, The Institute for Health Policy Studies, University of California. (iD) 0000-0002-2585-3996. H5: 17. Correo electrónico: jucarlosra@gmail.com 


\section{Introducción}

$\mathrm{C}_{\text {perpetuados socialmente, en los que prevalece el ideal de la masculinidad he- }}$ gemónica, considerada como un modelo estructurador de las relaciones entre sujetos de un grupo social. En este se reconocen características asignadas culturalmente entre las que resaltan ser heterosexual, fuerte, proveedor, protector, procrear, tener una familia, ser reconocido como jefe de la unidad familiar y, como consecuencia, gozar de privilegios en dicho contexto y en otros ámbitos sociales (Balam et al., 2018; Connell, 1995).

Sin embargo, estos elementos propios del modelo hegemónico no son permanentes; es decir, se modifican según los espacios, la temporalidad y las circunstancias en las que los sujetos se desempeñan, generando como resultado diferentes formas de configuración de las masculinidades.

A partir de esas características planteadas, en torno a esta forma de configuración del género de los hombres, es que se significa la paternidad, elemento constitutivo de la identidad masculina que, una vez establecida, permanece y es difícil de transformarse porque en el propio contexto existen elementos que favorecen su recreación (Botero et al., 2019). Si se parte de la existencia de variantes en la configuración de la masculinidad, esto trae como consecuencia diversas formas de significar y ejercer la paternidad (Scavino \& Batthyány, 2019).

Cuando la práctica de la paternidad ocurre en una edad considerada como precoz -como lo es la adolescencia- es importante contemplar las diversas situaciones a las cuales se enfrentan los sujetos en el marco de su cultura. Ejemplo de ello son los retos propios de la edad, tales como la búsqueda y afianzamiento de lo que es la identidad masculina (González-Flores \& Proano-López, 2017), sumado al cumplimiento de funciones esperadas y exigidas socialmente a los padres, como lo es la proveeduría y protección de su nuevo núcleo familiar. Adicional a esto, las dificultades relacionadas con políticas económicas y laborales, que no brindan los medios y garantías a los hombres adolescentes 
para obtener un trabajo con la remuneración adecuada que les permita dar cumplimiento a esos mandatos, según la construcción sociocultural de lo que es ser un hombre y, desde luego, un padre (De Martino, 2016).

Es común que, al hacer referencia a los padres adolescentes, se utilicen etiquetas y juicios por parte de sujetos con los que entran en contacto en la vida cotidiana, como es la familia de origen, los espacios de socialización y, de manera más amplia, la sociedad en general. A través de ellos se culpabiliza al hombre adolescente por la irresponsabilidad, generando con esto presiones para que estos individuos puedan asumir su condición de padre que, a pesar de ser algo esperado en la vida del hombre, se da de manera anticipada, llevando a que la transición de la niñez a la adultez sea más rápida y con mayores dificultades (Lopes et al., 2016; Salguero-Velásquez, 2014).

La paternidad en ese grupo etario es un tema poco abordado (De Martino, 2018). En los casos que se realiza está enmarcado en los estereotipos y la mirada predominante sobre el género de los hombres, que conlleva al desconocimiento de necesidades, expectativas e incluso emociones de estos sujetos adolescentes. Situación similar encontrada en el análisis de las interacciones parentofiliales, donde se ratifica que predominan las barreras y asignación de funciones según el sexo biológico (Aya-Angarita \& García-Suárez, 2020).

En muchos casos la carencia de información es subsanada con supuestos que no necesariamente se adecuan a la realidad en el ejercicio de la paternidad, considerándose como poco participativa, etiquetándose y haciendo señalamientos negativos que generan brechas y exclusiones para la vivencia en cada una de las etapas que implica la paternidad (Jayo, 2017).

Debido a estas situaciones y realidades del fenómeno de la paternidad temprana, se planteó la necesidad de reconocer que este es un rol que reviste distintas aristas; por lo que para este artículo se tomaron como preguntas guía: ¿cuál es el significado de la paternidad para hombres adolescentes residentes en la Zona Metropolitana de Guadalajara, México? ¿A partir de qué elementos contextuales y relaciones sociales se construye la paternidad en los hombres adolescentes participantes del estudio? Los anteriores interrogantes orientaron el objetivo de este artículo, el cual consistió en comprender la construcción y significados de la paternidad presente y activa de hombres adolescentes que residen en la Zona Metropolitana de Guadalajara, México, desde una perspectiva socioconstruccionista. 


\section{Método}

Este estudio corresponde a un diseño cualitativo interpretativista, para comprender los fenómenos que integran la perspectiva individual y colectiva de los participantes; ello con el fin de develar la realidad diversa presente en el campo (Maxwell, 2019), con una característica como lo es el escaso abordaje de experiencias, significados y emociones que condicionan la invisibilidad social de los hombres adolescentes en el ejercicio de la paternidad, más allá de los conceptos y funciones tradicionales (Parada-Rico \& GarcíaSuárez, 2017).

La perspectiva teórica utilizada fue el construccionismo social, el cual requiere la incorporación de elementos contextuales, culturales, la precisión del momento histórico y las experiencias colectivas para llegar a la comprensión de los significados y las prácticas en la vida cotidiana (De Jesús-Reyes \& Cabello-Garza, 2011). Este enfoque propone que los conceptos y significados que asumen los individuos son el resultado de procesos sociales, de la negociación de las intersubjetividades; es decir, que un significado es algo dinámico de acuerdo con aspectos temporales, espaciales, sociales y culturales y que está dado por la forma en cómo se comparten las creencias, actitudes y comportamientos en un grupo social. Por lo que se busca comprender la forma en cómo se producen y el uso que se les da a dichos significados, enmarcados en el contexto en que se desarrollan (Gergen \& Gergen, 2011).

En tal sentido, se hizo necesario analizar la manera en que las personas aplican su experiencia presente, su historia de vida, las influencias del contexto social y el conocimiento en un determinado tema, así como la negociación para llegar al consenso sobre la creación de un significado, para dar paso al conocimiento y prácticas socialmente aceptadas (Berger et al., 2001).

\section{Participantes}

En este estudio participaron 28 hombres, entre 16 y 19 años, residentes en Zona Metropolitana de Guadalajara, México, padres o que su pareja estaba en embarazo. El nivel socioeconómico fue bajo y medio; la escolaridad, en la mayoría de los casos, no superaba la preparatoria. Los lugares donde se encontraron a estos participantes fueron: en una institución de salud, en una organización no gubernamental y en instituciones de educación básica y media (secundaria y preparatoria). En la tabla 1 se presenta a los participantes 
(se utilizan seudónimos para preservar su anonimato), considerando si planearon o no la paternidad, sus edades y nivel de escolaridad.

\section{Tabla 1}

\section{Descripción sociodemográfica de los participantes}

\begin{tabular}{lcllcl}
\hline \multicolumn{3}{c}{ No planearon la paternidad (NP) } & & \multicolumn{2}{c}{ Planearon la paternidad (P) } \\
\hline \multicolumn{1}{c}{ Nombre } & Edad & Nivel de escolaridad & Nombre & Edad & Nivel de escolaridad \\
\hline Jorge Alejandro & 16 & Secundaria en curso & Edwin & 16 & Secundaria completa \\
Alonso & 16 & Preparatoria en curso & Cesar & 18 & Primaria completa \\
Llano & 17 & Preparatoria en curso & Derek & 19 & Preparatoria incompleta \\
Ezequiel & 18 & Preparatoria incompleta & Luis & 19 & Secundaria en curso \\
Eliceo & 19 & Secundaria incompleta & Francisco & 19 & Secundaria completa \\
Osvaldo & 19 & Preparatoria completa & Gerardo & 17 & Secundaria en curso \\
Romeo & 16 & Preparatoria en curso & Daniel & 17 & Preparatoria incompleta \\
Adrián & 16 & Primaria incompleta & Juan Manuel & 18 & Secundaria completa \\
Javier & 16 & Secundaria incompleta & José Manuel & 19 & preparatoria en curso \\
Patricio & 18 & Preparatoria en curso & Juan Carlos & 19 & Secundaria completa \\
Andrés & 19 & Preparatoria completa & Gilberto & 19 & Preparatoria completa \\
Luis Enrique & 19 & Preparatoria incompleta & Ernesto & 19 & Preparatoria completa \\
& & & Gonzalo & 17 & Preparatoria en curso \\
& & & Enrique & 17 & Secundaria completa \\
& & & Oliver & 18 & Secundaria completa \\
& & Alan & 19 & Preparatoria en curso \\
\hline
\end{tabular}

Para incluir a los participantes se tuvieron como criterios que tuvieran edad dentro del rango entre los 15 y 19 años, que estuvieran viviendo el rol de padres, sin distingo de etapa; esto significa que se incluyeron aquellos cuyas parejas estaban en embarazo, los padres recientes al momento de la entrevista y aquellos que ya eran padres con anterioridad.

Debido a las dificultades que representaba la localización de adolescentes con estas características, se identificaron personas clave que tuvieran acceso a población adolescente, como maestros, coordinadores, orientadores, directivas de distintas instituciones educativas y de salud. La incorporación de los participantes se hizo a través de un muestreo por conveniencia.

Es importante mencionar que todos los participantes ejercían la paternidad activa, que hacían frente a las responsabilidades de tener una pareja estable, un hijo/hija y las implicaciones de la formación de una familia. En este sentido, aquí se muestran las experiencias vividas por los padres adolescentes comprometidos con el ejercicio de la 
paternidad. Esta condición fue un hallazgo de la investigación y no un criterio de inclusión. El número de participantes fue definido a partir del muestreo teórico, utilizando el criterio de saturación de la información (García, 2015; Nascimento et al., 2018).

$\mathrm{Al}$ incluirse hombres que eran menores de edad, se contó con la aprobación de los padres o adultos responsables, quienes firmaron el consentimiento informado y el asentimiento por parte de los participantes. Para los mayores de edad, ellos aceptaron y firmaron el consentimiento informado. Adicional a esto, en el caso en el que los participantes hacían parte de instituciones educativas, se contó con la aprobación de los rectores.

\section{Recolección de la información}

Para la recolección de la información se tuvieron en cuenta las características del socioconstruccionismo, por lo que se utilizaron técnicas que privilegiaron el lenguaje, considerado como el medio a través del cual se constituye y se regula la realidad (ÍñiguezRueda, 2011) con estrategias metodológicas y analíticas basadas en el discurso (Gergen \& Gergen, 2011).

Se realizaron entrevistas semiestructuradas, con una guía construida a partir de la revisión de la literatura; además, se incluyeron temas emergentes y de relevancia para la comprensión del fenómeno de estudio. La guía de entrevista incluyó los siguientes temas: antecedentes personales y familiares, emociones, redes de apoyo, cambios percibidos en el presente y para el futuro, actitud frente a la paternidad, así como significados y prácticas de la masculinidad. Las entrevistas fueron individuales, en espacios asignados por las instituciones en que se encontraban los participantes. Las entrevistas se audiograbaron, previa autorización de los sujetos y de los adultos a cargo.

También se realizó observación participante a través de redes sociales (Facebook), reconociendo que estos espacios permiten diversas formas de socialización y prácticas que son significativas para las personas, en particular en este grupo de edad. Se creó una Fanpage (grupo cerrado) en la que se buscó identificar varios elementos: características del contexto cercano de los participantes, imágenes y comentarios que hacían sobre su hijo/hija y la pareja, las interacciones con los pares, siendo estos actores fundamentales para la aceptación, inclusión y reconocimiento en los grupos sociales con los que interactúan. Además de esto, se tuvo la posibilidad de hacer intercambios y diálogos a través del chat, lo que permitió tener un contacto frecuente para obtener información valiosa que contribuyera a la construcción y comprensión del fenómeno de interés, así como la triangulación de la información recabada durante el trabajo de campo. 
Aunque se procuró obtener la información en una sola sesión de entrevista, en los casos en que fue necesario profundizar o aclarar algunos temas abordados en ella, se acudió de nuevo a las instituciones en las cuales fueron ubicados inicialmente o vía Facebook.

\section{Análisis}

Para el análisis de la información se realizó la transcripción literal para garantizar el sentido de lo expresado por cada uno de los participantes (Bassi-Follari, 2015). Se organizó el corpus narrativo y se procedió a realizar la codificación e identificación de categorías preestablecidas a través de la revisión de la literatura, así como las emergentes en el proceso de análisis; esto último se realizó por medio del software ATLAS.ti (versión 7), con licencia de la Universidad de Guadalajara.

El análisis de contenido se llevó a cabo considerando el contexto para hacer inferencias sobre el discurso, tanto escrito (transcripción de entrevistas, conversaciones por redes sociales) como visual (fotografías y videos), que favorecieron la observación de hechos concretos, expresiones y lenguaje no verbal, que en conjunto brindaron una perspectiva más comprehensiva para cumplir el objetivo de investigación (Cárcamo, 2018; Urra-Medina \& Sandoval-Barrientos, 2018)

Se verificó la cobertura de todas las temáticas en el corpus. Se realizó la descripción del contenido del texto, buscando poner en evidencia los mensajes explícitos e implícitos, las semejanzas y las diferencias discursivas, resaltando su análisis de manera situada, para comprender los significados y prácticas de la paternidad por parte de los hombres adolescentes del estudio (Denzin \& Lincoln, 2012; Gomes, 2012). La validez de la información se garantizó a través de la triangulación de instrumentos; además, se hizo contrastación teórico metodológica y entre investigadores (Torres, 2021; Samaja, 2018).

\section{Resultados}

Para la presentación de los resultados y ejemplificación de las narrativas de los hombres participantes, se recurrió al uso de fragmentos de entrevistas; en estos se asignan nombres que corresponden a seudónimos, seguidos de la edad y las letras $\mathrm{PCu}$, para preparatoria en curso; PC, preparatoria completa; PI, preparatoria incompleta; SCu, secundaria en curso; SC, secundaria completa. 


\section{Conociendo a los padres adolescentes}

Partiendo de la premisa de la historicidad y relaciones sociales como elementos determinantes para la construcción de significados y prácticas sociales, se hizo necesario hacer una descripción de los participantes y su entorno social.

Con los participantes se formaron dos grupos emergentes a partir de la planeación de la paternidad: el primero es de 16 adolescentes quienes manifestaron que fue una decisión hablada y planeada en conjunto con su pareja, evidenciando así la participación y capacidad de decisión de estos hombres con respecto a su paternidad. De estos, ocho adolescentes manifestaron que fue la pareja la que hizo la propuesta de formar una familia, siendo un elemento importante porque, más que hablar de tener un hijo o hija, lo que quieren es formar la familia y lograr el reconocimiento social.

$\mathrm{Al}$ principio sí me dijo: ponte condón; pero ya después dijo: no, yo quiero ser mamá. Y yo le dije: no, pues yo papá, y pues ya no usamos condón. O sea, que los dos queríamos... Nosotros sí pensamos, hablamos y queremos, y pues ya nació mi hija. (Gonzalo, 17, PCu)

El segundo grupo fueron 12 que no planearon su paternidad; ellos sí deseaban tener un hijo, así que planeaban con sus parejas hacerlo a mediano o largo plazo, argumentando la importancia de terminar los estudios, mínimo tener la preparatoria (bachillerato) concluida y ser mayores de edad, para poder obtener un mejor trabajo y cumplir con las funciones de sostenimiento económico para garantizar una vida adecuada para su nueva familia. Esto refleja que, a pesar de ser una población joven, en la que se ha planteado resistencia a los modelos tradicionales de paternidad, no se logra desmantelar por completo, evidenciándose tanto en el discurso de los hombres como en las personas que están en el entorno (Salguero et al., 2020).

Los varones de este estudio tenían una relación activa con la pareja; sin embargo, la convivencia fue muy diversa. En el grupo de los que no planearon su paternidad, tres vivían únicamente con la pareja; el resto carecía de recursos para formar su núcleo familiar y, por tanto, vivían cada uno con su familia de origen (seis) o vivían en casa de la familia de la pareja (tres). En el grupo de los que sí planearon la paternidad, cuatro vivían con sus respectivas familias de origen; cinco solo con la pareja; otros cinco con la pareja y la familia de ella y uno con la pareja y la familia de él; este último dio el siguiente argumento: «Porque siento y sentimos los dos que era mejor que el hombre se llevara a la mujer, no que la mujer al hombre. Entonces, ahí se vería raro» (Daniel, 17, PI). 


\section{Paternidad y su significado}

¿Qué significa para estos varones la paternidad? Lo primero que sale a relucir es el deber ser, el ideal de padre: el que siempre está presente, apoya, comprende, brinda y recibe amor, pero también el encargado de proporcionar sustento económico. Es decir, se refuerza el ideal del padre tradicional con respecto a funciones de proveeduría, posición de privilegio, dando la imagen positiva de este rol y destacando la importancia del aspecto emocional y cercanía con la familia (Montenegro et al., 2019).

En la narrativa de los varones se plantea la intención de ejercer una paternidad consciente de sus deberes y derechos con respecto a los hijos/hijas; ejercer una paternidad presente y corresponsable que permita mayor expresividad y afectividad. Sin embargo, como se mencionó anteriormente, estas ideas hacen parte del deber ser, en el que, a pesar de que existe una propuesta de cambio, persisten roles tradicionales al interior de las familias. Ello se ha relacionado con características socioeconómicas, donde padres con mayores dificultades económicas mantienen roles tradicionales a causa de la falta de oportunidades que disminuye el tiempo para compartir en familia (Hernández-Quirama et al., 2021).

La experiencia que estos padres revela diversas dificultades, presiones sociales, económicas y familiares, pero que, en contraste, les permite llenar vacíos que tuvieron a lo largo de su infancia, así como la satisfacción de responder ante las diferentes situaciones y necesidades:

Mi papá nunca estuvo casi con nosotros por su trabajo. Mi papá no más era de que oyes me pidieron esto en la escuela, ahí te va; y ahora de grandes se lo echamos en cara y dice: ¿Qué les hizo falta cabrón? No, pos nada, no. Y yo no quiero eso para mi niña o mi niño. Por eso ya ahora mejor busco un trabajo donde pase el tiempo con los dos. Más que un padre, ser amigo porque fue algo que yo no tuve y siempre lo soñé. (Luis, 19, SCu)

Por otro lado, Gonzalo (17, Pcu) anota: «Todos los maestros me han dicho eso, que soy bien valiente pa tener una niña a esta edad; que hay unos que tienen la niña y las arrumban ahí y no les ponen importancia. Entonces, me han dicho que yo soy diferente.»

\section{Actores sociales como ejemplo de comportamiento y red de apoyo para la construcción de la paternidad}

¿Quién o qué moldea el significado y el ejercicio de la paternidad? Para los varones, su propia experiencia fue fundamental para ir dando forma a su desempeño como padres; pero aquello que es central para esta construcción es la relación con la figura paterna 
(Ladino \& López, 2018), destacando las experiencias positivas y negativas como referencia para ejercer este rol en la sociedad, la manera como quieren conducirse por la vida y comportarse con sus hijos/hijas. Ello se pudo evidenciar a través de la narrativa, tanto de los que ya eran padres como de los que estaban por serlo.

Existen casos que demuestran cómo una mala experiencia y un mal ejemplo motivan a cambiar y a no repetir esos patrones de comportamiento, entre los cuales está el abandono. Alonso, Eliceo, Osvaldo y Luis Enrique son hijos de padres separados, incluso reportan no conocer a su padre biológico. Todos coinciden en que no quieren dejar a su hijo solo; no quieren que pase por lo mismo que ellos pasaron. «Yo no quiero que él (su hijo) esté como yo estuve, así como..., ¡desprotegido! Sin nadie, ni padre ni madre ni nadie; era muy rechazado yo» (Eliceo, 18, SI).

Por el contrario, están los casos en los que definitivamente su ejemplo a seguir y repetir es su papá y la relación entre la familia y el apoyo constante que les brindan:

Bueno, pues mi padre fue un gran ejemplo... No pues, qué padre que mi papá me haya dado toda esa oportunidad y te dan ganas de ser como él. Porque como tú ves que él es muy bueno, es muy bueno conmigo. Tengo que agarrar algo de él porque pues es un gran, es una gran persona... Lo admiro mucho; es muy trabajador, da consejos; pues si estás en un problema o cosas así, te dice: no, pues yo te recomiendo que hagas pues así. Pues da, ¿cómo te podría decir? Tiene una personalidad muy agradable y es muy paciente. (Patricio, 18, $\mathrm{PCu}$ )

Varios participantes tuvieron la presencia de la figura paterna en sus vidas. Sin embargo, cuestionan mucho la función de estos al no compartir y no darles cariño por estar trabajando; de manera que algo que desean estos varones es poder tener un trabajo que les permita tener una buena remuneración y, además, el tiempo suficiente para poder interactuar con sus hijos/hijas:

Pues mucho mejor que mis padres, porque, como me crió mi papá, no me quejo...; pero como mi papá nunca estuvo casi con nosotros por su trabajo, pues para mí, mi mamá; y mi papá pues es mi mamá, porque ella siempre estuvo al pie del cañón. (Luis, 17, $\mathrm{SCu}$ )

La construcción del significado de la paternidad en estos hombres es el resultado de múltiples elementos sociales y culturales, así como de la propia experiencia, a partir de la cual hay un proceso de asociación con sus conocimientos previos, las vivencias, el sistema de creencias y valores, así como sus realidades; lo que finalmente configura su ejercicio de paternidad: 
No, pues yo trato, yo digo que más o menos uno se basa también en nuestros padres, ¿̨o? Más o menos en crianza. Pos yo trato de darle lo mejor que pueda, de educarla bien. Bueno, pos de enseñarle cosas pa que al rato no se hagan unas personas que no..., bueno, que no te saquen los ojos al rato; educarlos bien porque o si no son muy malcriados. (Derek, 19, PI)

\section{Paternidad: motivaciones y decisiones}

Fueron diversas las razones o motivos por los cuales varios varones tomaron la decisión de ser padres. Entre estas resaltan las características de la relación con la pareja, pensando en la seguridad y estabilidad que percibían, sintiéndose deseosos de formar una familia con ella y poder tener un hijo/hija, reconociendo la importancia de la formación de la familia, debido a que así podían confirmar su identidad como hombres (Botero et al., 2019), logrando reconocimiento social y, en muchos casos, supliendo las carencias percibidas desde el núcleo familiar, principalmente el cariño (Botero \& Castrillón, 2015), tanto para demostrarlo como para recibirlo.

A diferencia de lo que se espera socialmente como resultado de los estereotipos asignados al género de los hombres, en este grupo de participantes muchas de las parejas se encargaron de tomar la iniciativa de formar una familia; situaciones en las que los varones en ningún momento demostraron una respuesta negativa. Manifestaron que después de aceptar había momentos en que se arrepentían; pero no era algo que le transmitieran a su pareja. Ello se puede analizar desde los estereotipos de las masculinidades, donde es evidente que más vale callar y seguir adelante, que demostrar arrepentimiento, miedo o cualquier signo de debilidad.

Varios decían que quisieron porque era algo que deseaban experimentar, el saber lo que es tener un hijo/hija y saber lo que vivieron sus padres, además de asumir responsabilidades y tener la satisfacción de cumplir ante esos mandatos. Esto ratifica la idea de que no es el simple hecho de ser padre, sino también poder cumplir con las obligaciones que esto conlleva; lo anterior, a pesar de ser tan jóvenes y de que enfrentan dificultades propias de su etapa del ciclo vital, de que la estructura social que no les es favorable para el ejercicio de una paternidad precoz, entre otras. La sociedad no tiene previsto la inclusión de estos sujetos en la vida laboral, así que forman parte de la cohorte etárea que enfrenta la mayor proporción de desempleo (Ruiz \& Ordaz, 2011)

Otra de las motivaciones que se encontraron, y sobre las cuales se quiere llamar la atención de manera especial, es que fue considerada como la manera de «ponerse en paz». Pero ¿a qué se referían con esto? Muchos explicaron que formaban parte de grupos 
o pandillas en las que consumían alcohol, cigarrillo o algún tipo de sustancia psicoactivas, ejercían conductas violentas, enfrentamientos con otros, como parte de la dinámica del grupo al que pertenecían, y tuvieron como resultado lesiones que identificaron como algo muy grave. Condiciones que en conjunto les impulsaban a buscar la manera de cambiar. Otros simplemente tenían un gusto por la fiesta y estar con los amigos.

Todos manifestaron que fue a partir de la convivencia con la pareja o a la noticia del embarazo que decidieron cambiar; esto coincide con los hallazgos de Fuller (2000) en la población masculina de Perú, en la que se dan cambios en las metas, sueños y en la relación con sus pares, así como cambios de comportamiento.

Los cambios en las actitudes son un resultado multifactorial que ha sido abordado en varios estudios, empezando con las ideas asociadas a la masculinidad y lo que representa ser padre dentro de la construcción de la identidad del ser hombre (Deavila-Pertuz, 2015) en la que cumplir con características como es ser guía, protector y, además, el cambio de estatus de hombre sin responsabilidad al hombre padre y jefe de familia son las principales motivaciones para mejorar sus comportamientos (Álvarez-Vivar et al., 2017; Villegas, 2005). El tener una relación de pareja estable (lo cual se consolida a través de los hijos/hijas) es otro elemento motivacional para dejar comportamientos de riesgo, en los que se piensa principalmente en no defraudar a su descendencia.

Para estos adolescentes es claro el riesgo inminente al que están expuestos, desde lesiones (muchos lo expusieron en sus narrativas), la muerte o ir a la cárcel. Se podría pensar que la paternidad y la vida en pareja es el motivo de desistimiento de actos delincuenciales y la mejor razón para dejar las pandillas; evidenciando cómo los significados atribuidos al género de los hombres moldean su comportamiento, teniendo en cuenta los mandatos sociales sobre la proveeduría, la formación de la familia y el padre como ejemplo de comportamiento (Álvarez-Vivar et al., 2017); como lo narra Oliver, para quien fue sinónimo de salvación ante sus malos comportamientos:

Porque yo ya andaba jodido; bueno, por la calle. Yo me portaba mal y me sucedieron muchas cosas y dije: no tengo que...; fui a psicología y así leve, no me gustó mucho, pues no me gusta a mí tanta la terapia de eso, y dije: me tengo que calmar. (Oliver, 18, SI) 


\section{Discusión}

En este estudio entre hombres adolescentes, las narrativas sobre el significado de la paternidad y la práctica reflejan el deber ser, en el que manifiestan de manera explícita que el ejercicio de la paternidad lo construyen a partir de la resistencia a señalamientos, mandatos asociados al género y los roles diferenciales según el sexo biológico. Buscan demostrar que sobrepasan las atribuciones relacionadas con su edad como la inmadurez y el error ante la situación vivida. Esto se ha documentado en otros estudios, resaltando aspectos positivos de la propia vivencia de la paternidad precoz, desde la formación de un nuevo núcleo familiar hasta el involucramiento en el proceso de crianza (Botero \& Castrillón, 2015).

Estas respuestas obedecen a una situación ideal que plantean como intención; un sueño, un anhelo en el ejercicio de una paternidad particular. Sin embargo, se evidencian situaciones diversas en la significación de este proceso, mostrándose una heterogeneidad de experiencias y, por lo tanto, de las prácticas de estos padres adolescentes.

En este sentido, hay un punto de convergencia entre este estudio y otros frente a las características que pueden determinar las experiencias y, por ende, la forma de significar la paternidad; entre estas se destacan la edad, la condición social, las características de las relaciones, ya sea de pareja o con la familia, teniendo en cuenta la importancia de estas para el proceso de adaptación y contención emocional. Por otro lado, la etapa que estaban viviendo, es decir, si ya había nacido el hijo/hija o estaba en proceso el embarazo (Parada-Rico \& García-Suárez, 2017).

A pesar de la diversidad de prácticas y experiencias, emergieron elementos comunes en estos adolescentes: la persistencia de los modelos tradicionales asociados a la masculinidad como punto determinante para definir lo que es ser padre y cómo ejercer dicho rol. Se resalta la distribución de tareas para los hombres tales como el sostenimiento económico y la protección de la familia como un elemento motivacional y significativo tanto en el proceso como en las experiencias que genera este rol en los hombres adolescentes (Salguero et al., 2020). Cabe destacar que estos significados y formas de desempeñarse no son exclusivas de la población adolescente. Esto se evidencia en otros estudios realizados en padres ante situaciones críticas como lo es la necesidad de tener a su hijo/hija en una unidad de cuidados intensivos, llevando a situaciones extremas que generan procesos de trabajo emocional como estrategia de contención, sumado a la idea del cumplimiento de mandatos sociales y que, en contraste, hay un cuestionamiento y una clara 
manifestación de acercamientos hacia la corresponsabilidad y hacia búsqueda de igualdad con la pareja (Flores \& Galindo, 2018; Izquierdo-Sánchez \& Ferrer-Ribot, 2018), como ocurre con los participantes de este estudio.

Es decir, si bien existe una resistencia a modelos tradicionales, en realidad, y como consecuencia de la estructura social y económica en la que están inmersos estos adolescentes, mantienen prácticas que a lo largo de la historia se han dado como consecuencia de la división sexual del trabajo, por ejemplo, el que las labores en espacios públicos dificultan la socialización y disminuyen el tiempo para compartir con la familia (FigueroaPerea \& Franzoni, 2011; Olavarría, 2017).

Por otro lado, están aquellos que tienen mayor participación en cada una de las etapas de su breve experiencia de paternidad. Tal como se ha encontrado en otras investigaciones, se caracterizan por tener mayor apoyo de la familia, facilitando el cumplimiento de sueños, proyectos de vida e incluso responder a los mandatos, lo que les permite demostrar relaciones cercanas, activas y más emocionales (Hernández-Quirama et al., 2021).

En esta población persisten elementos motivacionales enmarcados en lo que implica el género de los hombres y la construcción de la identidad masculina, en la que se está en la búsqueda permanente de demostrar y mantener atributos propios de estos sujetos (reconocimiento social y reproducción), en el que ser padre jerarquiza a los varones y les permite la comprobación de su capacidad de hacerse responsable de otros (en este caso de su hijo/hija, de la pareja y, desde luego, de sí mismos; Rojas, 2021).

También se pudo evidenciar que en la mayoría de las situaciones hay una fuerte motivación, desde el punto de vista emocional, para la formación de una familia: suplir carencias experimentadas durante la infancia, tales como el abandono físico y emocional por parte del padre (en la mayoría de los casos) o por relaciones difíciles en el núcleo familiar.

Otra motivación es la modificación de conductas negativas que ponían en peligro sus vidas, viendo en su condición de padres la opción para cambiar comportamientos de riesgo; siempre con la idea de que querían vivir más para ver a sus hijos/hijas crecer, para ser un guía y ejemplo en la vida de estos (Álvarez-Vivar et al., 2017). Es decir, que la paternidad fue una experiencia determinante para transformar, dar sentido y direccionar sus vidas.

A partir de estos hallazgos, se concluye que el significado de paternidad para estos hombres se basa en la calidad de la relación y experiencias propias con su familia, la figura paterna, así como la posibilidad de cumplir su proyecto de vida y mandatos sociales asignados a esta figura. Para estos hombres adolescentes, en muchos de los casos, su hijo/ 
hija y pareja representan la posibilidad de tener una familia, apoyo y cariño, así como el tener control y dirección de sus vidas para ser más que el proveedor económico: ser el ejemplo, guía para su familia y llegar al tan anhelado reconocimiento social.

Hay un avance hacia una corresponsabilidad en el proceso de paternidad; sin embargo, todavía falta recorrer el camino y lograr que estos hombres sean visibilizados y que las políticas públicas, económicas y laborales consideren la perspectiva y necesidades de estos grupos etarios para que sus condiciones sean mejoradas y lograr cumplir con sus responsabilidades y construir una paternidad más activa, cercana y corresponsable.

Con los hallazgos presentados en este artículo se aporta una visión desde las perspectiva de los hombres, lo cual permite orientar nuevas investigaciones sobre una temática de interés general como lo es el embarazo/paternidad adolescente en la sociedad actual. Así, se demuestra la importancia de incluir a todos los actores para tener una comprensión integral de las experiencias, expectativas, los proyectos de vida; así como el impacto que tiene el embarazo/paternidad a temprana edad, debido a la posibilidad de cumplir sueños, lograr reconocimiento y estatus dentro de un grupo social.

Se pudo dar cuenta de los significados y prácticas sobre la paternidad en un grupo de hombres adolescentes residentes en la Zona Metropolitana de Guadalajara, permitiendo contrastar las fronteras de los estereotipos en el marco de una categoría analítica como es el género de los hombres, develando aspectos vivenciales, contextuales e históricos que determinan el ejercicio de la paternidad. Sin embargo, se tuvo la limitante de no tener acceso a aquellos hombres que no tuvieron una participación activa en cada una de las etapas que implica la paternidad; es decir, no se pudo escuchar la perspectiva de aquellos padres adolescentes ausentes, lo cual sería importante y necesario para tener una comprensión mayor de este fenómeno.

\section{Agradecimientos}

Agradecemos al Dr. Alexis Chávez Díaz por su apoyo y acompañamiento en el proceso de trabajo de campo para poder acceder a los diferentes espacios y llevar a buen fin el proyecto de investigación. 


\section{Referencias}

Álvarez-Vivar, L., Bustamante-Cárcamo, Y., \& Salazar-Muñoz, M. (2017). Paternidad y su incidencia en el desistimiento delictual: una revisión teórica. Revista Criminalidad, 59(1), 65-75.

Aya-Angarita, S. L., \& García-Suárez, C. I. (2020). El universo emocional en las interacciones parentofiliales: un acercamiento al estado del arte. Revista Latinoamericana de Ciencias Sociales, Niñez y Juventud, 18(1), 1-17. https://doi.org/g6gr

Balam, J. M., Valdes, A. G., Ramírez, A., \& Padilla, N. (2018). Significado psicológico, roles y expectativas de la paternidad en adolescentes mexicanos: estudio exploratorio. Psicología Iberoamericana, 26(1), 42-52. https://doi.org/10.48102/pi.v2611.32

Bassi-Follari, J. E. (2015). El código de transcripción de Gail Jefferson: adaptación para las ciencias sociales. Quaderns de Psicologia, $17(1)$, 39-62. https://doi.org/dswp

Berger, P. L., Luckmann, T., Zuleta, S., \& Berger, P. L. (2001). La construcción social de la realidad. Random House.

Botero, L. D., \& Castrillón, L. C. (2015). La experiencia de la paternidad en adolescentes. Revista Virtual Universidad Católica del Norte, 3(46), 89-101.

Botero, L. D., Hernández, J. C., \& Caicedo, J. (2019). Configuración de la identidad de padres adolescentes: un estudio cualitativo. Revista Colombiana de Ciencias Sociales, 10(2), 377-398. https://doi.org/10.21501/22161201.2776

Cárcamo, B. (2018). El análisis del discurso multimodal: una comparación de propuestas metodológicas. Forma y Función, 31(2), 145-173. https://doi.org/g695

Connell, R. (1995). Masculinidades. Polity Press.

Deavila-Pertuz, L. (2015). Paternidades jóvenes en los arrabales cartageneros: Análisis de las paternidades en jóvenes hombres en las edades de 21 a 23 años en Cartagena de Indias, caso unidad comunera de Gobierno 6, 2015. [Tesis de grado]. Universidad de Cartagena.

De Jesús-Reyes, D., \& Cabello-Garza, M. L. (2011). Paternidad adolescente y transición a la adultez: una mirada cualitativa en un contexto de marginación social. Iberóforum. Revista de Ciencias Sociales de la Universidad Iberoamericana, 6(11), 1-27.

De Martino, M. S. (2016). Padres adolescentes y jóvenes: debates y tensiones. Revista Katálysis, 19(1), 91-99. https://doi.org/10.1590/1414-49802016.00100010

De Martino, M. S. (2018). Ser padres y madres a edades tempranas: experiencias y significados. Revista Prisma Social, (23), 209-234. 
Denzin, N. K., \& Lincoln, Y. (2012). La investigación cualitativa como disciplina y como práctica: manual de investigación cualitativa. Gedisa

Figueroa-Perea, J. G., \& Franzoni, J. (2011). Del hombre proveedor al hombre emocional: construyendo nuevos significados de la masculinidad entre varones mexicanos. En F. Aguayo, \& M. Sadler (Eds.), Masculinidades y políticas públicas: involucrando hombres en la equidad de género (pp. 64-82). Universidad de Chile.

Flores, A., \& Galindo, E. A. (2018). Significados de paternidad y maternidad en parejas en transición en Tlaxcala, México. Revista Rumbos TS. Un espacio crítico para la reflexión en Ciencias Sociales, (18), 67-93.

Fuller, N. (2000). Significados y prácticas de paternidad entre varones urbanos del Perú. En Paternidades en América Latina (pp. 35-9o). Fondo Editorial de la Pontificia Universidad Católica del Perú.

García, M. (2015). Aspectos metodológicos de la investigación cualitativa. En L. Abero (Ed.), Investigación educativa: abriendo puertas al conocimiento (pp. 101-118). Consejo Latinoamericano de Ciencias Sociales.

Gergen, K., \& Gergen, M. (2011). Reflexiones sobre la construcción social. Paidós.

Gomes, R. (2012). El análisis de datos en la investigación cualitativa. En M. C. De Souza (Coord.), Investigación social: teoría, método y creatividad (pp. 53-64). Lugar Editorial.

González-Flores, D. G. R., \& Proano-López, E. L. (2017). La influencia de los pares y la familia en el inicio de la actividad sexual en adolescentes. Augusto Guzzo Revista Acadêmica, 1(19), 39-53.

Hernández-Quirama, A., Rivero-Rubio, C., \& Linares-García, J. (2021). Significados de paternidad para jóvenes universitarios: cotidianidad, relaciones y cambios en proyecto de vida. Revista Latinoamericana de Ciencias Sociales, Niñez y Juventud, 19(1), 1-21. https://doi.org/10.1160o/rlcsnj.19.1.3924

Íñiguez-Rueda, L. (2011). Análisis del discurso: manual para las Ciencias Sociales. UOC. Izquierdo-Sánchez, L., \& Ferrer-Ribot, M. (2018). Las experiencias del padre en las Ucin: una revisión desde la perspectiva de género. Revista Latinoamericana de Ciencias Sociales, Niñez y Juventud, 16(1), 55-69. https://doi.org/10.11600/1692715x.16102 Jayo, L. (2017). Paternidad adolescente: una corresponsabilidad invisibilizada. Revista Puce, (105), 225-243. https://doi.org/10.26807/revpuce.voio.97

Ladino, N. I., \& López, P. Y. (2018). «Así hemos sido padres...: un aporte a la construcción de las representaciones sociales sobre paternidad». Pensamiento Americano, 11(20). https://doi.org/10.21803/pensam.v11i20.22 
Lopes, A. C. L., Könzgen, S. M., Schwartz, E., Netto de Oliveira, A. M., Corrêa, M., \& Jardim, V. M. da R. (2016). Percepções de homens sobre a vivência da paternidade na adolescência: Uma perspectiva bioecológica. Revista Gaúcha de Enfermagem, 37(1), e54692. https://doi.org/10.1590/1983-1447.2016.01.54692

Maxwell, J. A. (2019). Diseño de investigación cualitativa. Gedisa.

Montenegro, J. L., Orcasita, L. T., Tunubala, L. A., \& Zapata, L. J. (2019). Representaciones sociales sobre masculinidad y paternidad en padres con hijos gays. Investigación en Enfermería: Imagen y Desarrollo, 21(1), 1-22. https://doi.org/g699

Nascimento, L. C., Souza, T. V. de, Oliveira, I. C. dos S., Moraes, J. R. M. M. de, Aguiar, R. C. B. de, \& Silva, L. F. da. (2018). Theoretical saturation in qualitative research: An experience report in interview with schoolchildren. Revista Brasileira de Enfermagem, 71(1), 228-233. https://doi.org/10.1590/0034-7167-2016-0616

Olavarría, J. (2017). Trabajo, familia y género: precarización, y sindicalización en jefes/as de hogar de familias conyugales en Chile actual. Descentrada, 1(2), eо23-eo23.

Parada-Rico, D. A., \& García-Suárez, C. I. (2017). Padres y madres adolescentes en el ejercicio de la crianza. Revista Ciencia y Cuidado, 14(2), 113-129. https://doi.org/g7bb

Rojas, O. L. (2021). Masculinidades, desigualdad social y embarazo en varones adolescentes mexicanos. Revista Interdisciplinaria de Estudios de Género de El Colegio de México, 7, 1-34. https://doi.org/10.24201/reg.v7i1.817

Ruiz, P., \& Ordaz, J. L. (2011). Evolución reciente del empleo y el desempleo en México. Economía Unam, 8(23), 91-105.

Salguero-Velásquez, M. A. (2014). Formar familia, ser padre y estudiante universitario. Universidad Autónoma de Nuevo León, 6(29), 7-12.

Salguero, M. A., Yoseff, J. J., Soriano, M., \& Delabra, B. (2020). Presencias y ausencias paternas: la experiencia de hombres en Ciudad de México. Encrucijadas. Revista Crítica de Ciencias Sociales, 18, a1804.

Samaja, J. (2018). La triangulación metodológica (pasos para una comprensión dialéctica de la combinación de métodos). Revista Cubana de Salud Pública, 44(2), 431-443.

Scavino, S., \& Batthyány, K. (2019). Caminos hacia la corresponsabilidad: los varones en el cuidado infantil en Uruguay. Cadernos Pagu, (56). https://doi.org/g7bp

Torres, A. E. (2021). El transitar en la investigación cualitativa: un acercamiento a la triangulación. Revista Scientific, 6(20), 275-295. https://doi.org/g7bk

Urra-Medina, E., \& Sandoval-Barrientos, S. (2018). El análisis del discurso crítico en las investigaciones de salud. Enfermería Universitaria, 15(2), 199-211. https://doi.org/g7b3 Villegas, F. (2005). Las pandillas juveniles de Lima. Espacio Abierto, 14(1), 73-95. 